\title{
Methods for Ellipse Detection from Edge Maps of Real Images
}

\author{
Dilip K. Prasad ${ }^{1}$ and Maylor K.H. Leung 2 \\ ${ }^{1}$ Nanyang Technological University \\ ${ }^{2}$ Universiti Tunku Abdul Rahman (Kampar) \\ 1Singapore \\ ${ }^{2}$ Malaysia
}

\section{Introduction}

Detecting geometric shapes like ellipses from real images have many potential applications. Some examples include pupil tracking, detecting spherical or ellipsoidal objects like fruits, pebbles, golf balls, etc. from a scene for robotic applications, detecting ellipsoidal objects in underwater images, detecting fetal heads, cells, or nuclei in biological and biomedical images, identifying the eddy currents and zones using oceanic images, forming structural descriptors for objects and faces, traffic sign interpretation ${ }^{1}$, etc.

Given the very wide scope of applications, it is important that the ellipses can be detected from real images with high reliability. Specifically, it is important that the structures that are elliptical are indeed detected and the structures that are non-elliptical are not detected as

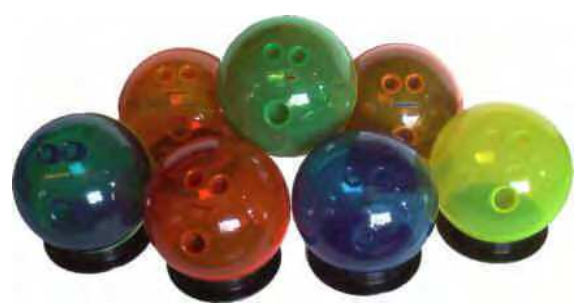

(a) an example of real image

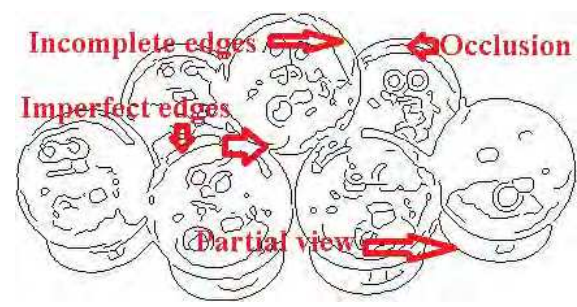

(b) its edge map (after histogram equalization and Canny edge detection)

Fig. 1. An example of a real image and the problems in detection of ellipses in real images

\footnotetext{
${ }^{1}$ (Antonaros and Petrou, 2001; Belaroussi et al., 2005; Bell et al., 2006; Burrill et al., 1996; Chia et al., 2009; Dijkers et al., 2005; Fernandes, 2009; Feyaerts et al., 2001; Foresti, 2002; Foresti et al., 2005; Fu and Huang, 1995; He et al., 2009; Hua et al., 2007; Hwang et al., 2006; Iles et al., 2007; Ji et al., 1999; Kayikcioglu et al., 2000; Kumar et al., 2009; Kuno et al., 1991; Liu et al., 2007; Lu et al., 2005; Matson et al., 1970; O'Leary et al., 2005; Prasad 2011c; Rosin and West, 1992; Salas et al., 2006; Shen et al., 2009; Shih et al., 2008; Smereka and Glab, 2006; Soetedjo and Yamada, 2005; Sood et al., 2005; Takimoto et al., 2004; Tang et al., 2000; Wang et al., 2006; Wu and Wang, 1993; Yuasa et al., 2004; Zaim et al., 2006; Zhang et al., 2003; Zhou and Shen, 2003; Zhou et al., 2009)
} 
ellipses. However, the problem of detecting ellipses in real images is very challenging due to many reasons. Consider the example given in Fig. 1.

Besides the obvious and ubiquitous presence of the problem of digitization in any digital image, there are other problems as seen in Fig. 1. The various challenges are summarized below succinctly:

1. Digitization: In digital images, the elliptic curves get digitized. As a consequence of digitization, the ellipses cannot be retrieved correctly and only an estimate of the parameters of the ellipses can be retrieved (Prasad and Leung, 2010b).

2. Presence of incomplete ellipses due to overlap and occlusion: One issue that can be easily envisaged in real images is that objects are usually present in overlap with each other, see the labels in Fig. 1(b) for examples. If the overlapping object is transparent or translucent, the boundaries of overlapped objects might still be available in the edge map as small disconnected edges (obtained after edge detection), see Fig. 2(a,c). However, if the overlapping object is opaque, the overlapped object is occluded and its incomplete boundary will appear in the edge map, see Fig. 2(b,d). If an image is cluttered by various objects of such nature, the problem gets very complicated to handle as such scenario results in various incomplete small edges in the image.

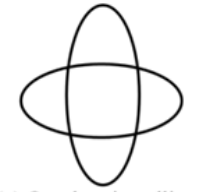

(a) Overlapping ellipses



(horizontal)

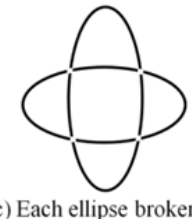

into 4 edges

(corresponding to (a))

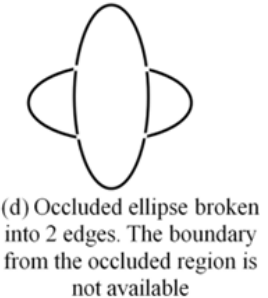

not available

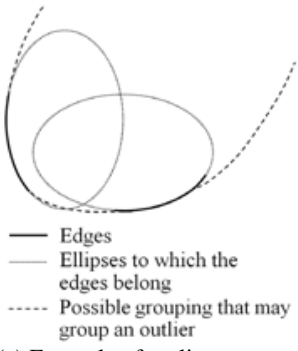

(e) Example of outliers

Fig. 2. Illustration of the presence of overlapping ellipses, occluded ellipses, and outliers

3. Presence of outliers: In cluttered background, some curved edges that are non-elliptic, may appear as if they are part of some elliptic edge. An example is shown in Fig. 2(e). The presence of such edges, referred to as the outliers, often results in false ellipse detections and degrades the performance of ellipse detection algorithms. Due to this reason, the incorrectly detected ellipses need to be filtered out at the end of the ellipse detection method.

4. Corruption in the quality of edge: Another problem that is encountered in real images is the deterioration of the boundary of the edge map due to the light and shadow conditions and the perspective of the object. Under different lighting conditions, boundaries in some region may be emphasized and appear sharp and clear, while boundaries in other regions might blur and deteriorate the edge in that region. Shadow effect may blur the region in which the boundaries of two objects overlap. Due to this, the boundaries of two objects may merge and appear to be smooth. Further, noise can appear in image due to imperfect imaging instruments and other external conditions like fog, glare, etc. Noise corrupts the quality of edge, rendering it to be non-smooth over small section of edge, and abrupt breaks in the boundaries. One such example can be found in Fig. 3. 


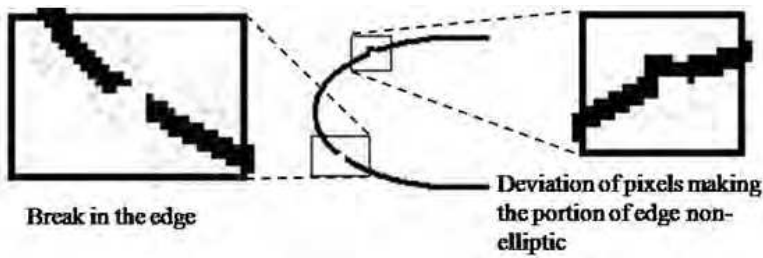

(a) Corruption of edges due to noise

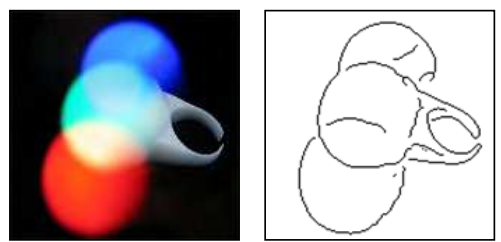

(b) Elliptic shapes look non-elliptic in edgemap due to light and shadow effect

Fig. 3. Illustration of the corruption in the quality of edges

5. Lack of a priori information: Another aspect of the considered problem is that no a priori information is available. It might have helped if the expected number of elliptic shapes, expected size of ellipses, or expected regions of focus were known a priori. However, real images may vary greatly in the scale and content, and in general such a priori information cannot be generated reliably.

\section{Contemporary ellipse detection methods}

Extraction of elliptic shapes from images has captured the interest of researchers for a long time. For two decades, numerous researchers are working on this problem. Many methods have been proposed for ellipse extraction in real images. The methods used for ellipse detection can be primarily categorized into four categories, viz., Hough transform (HT) based methods, least squares based methods, genetic algorithms based methods, and hybrid ellipse detection methods.

Hough Transform (HT) and its adaptations are widely used in many ellipse detection methods (Lu and Tan, 2008; McLaughlin, 1998; McLaughlin and Alder, 1998; Yuen et al., 1989). The key advantage of HT is that it does not require perfect connectivity of all the edge pixels belong to an ellipse and which makes it useful for ellipse detection in noisy and cluttered images compared to edge following based ellipse detection method. However, since the ellipse detection problem involves 5-dimensional parametric space in HT (Illingworth and Kittler, 1988). HT turns out to be a computation intensive method, requiring huge computation time and memory. In the last two decades, the researchers using HT to detect elliptical and circular segments have focused on providing computationally more efficient and faster HT adaptations. Two popular approaches used by researchers are dimensionality reduction in parametric space (Aguado et al., 1995; Aguado et al., 1996; Goneid et al., 1997; Ser and Siu, 1995; Yip et al., 1992; Yip et al., 1995; Yuen et al., 1989) and piecewise linear approximation of curved segments (Yip et al., 1992; Yip et al., 1995).

Least squares based methods usually cast the ellipse fitting problem into a constrained matrix equation in which the solution should give least squares error. From the mathematical perspective, important work for ellipse detection has been done by (Fitzgibbon et al., 1999; Rosin, 1993b; Rosin and West, 1995; Tsuji and Matsumoto, 1978). In terms of application, some interesting works include (Ellis et al., 1992; Kim et al., 2002; Meer et al., 1991). Fitzgibbon (Fitzgibbon et al., 1999) proposed a very robust and efficient least squares method for ellipse detection. This method is invariant to affine transformation and 
is computationally efficient. While Fitzgibbon (Fitzgibbon et al., 1999) is arguably the most popular method used in the image processing community, some further work in this direction has been done (Harker et al., 2008; Maini, 2006; O'Leary et al., 2005). We also note the family of work (Ahn and Rauh, 1999; Ahn et al., 2002; Ahn et al., 2001) which uses geometric properties within the least squares method. However, they still require non-linear constrained optimization and are not specific to ellipses. We emphasize here that though some work has been done for fitting ellipses to a cluster of data, they may not be specifically useful in detecting ellipses without suitable modifications.

Genetic algorithms are good at dealing with non-linear optimization problems in which there are many local minima. However, these algorithms are generally computation intensive and require a lot of time for convergence. The stochastic nature of such algorithms make them time consuming. Further, the possibility of premature saturation cannot be fully excluded in most cases and the algorithms have to be carefully designed for each problem. Some interesting adaptations of genetic algorithm for the ellipse detection (Kasemir and Betzler, 2003; Kawaguchi and Nagata, 1998a; Kawaguchi and Nagata, 1998b; Procter and Illingworth, 1994).

The final category is the hybrid ellipse detection methods (Chia et al., 2011; Chia et al., 2008; Kim et al., 2002; Mai et al., 2008; Prasad and Leung, 2010a; Prasad and Leung, 2010c). The hybrid ellipse detection methods use one or more of the above approaches as just an intermediate step in the ellipse detection algorithm. Other steps like sophisticated digital curve pre-processing techniques (Bhowmick and Bhattacharya, 2007; Carmona-Poyato et al., 2010; Masood, 2008; Prasad and Leung, 2010d; Prasad et al., 2011b; Prasad et al., 2012), curvature estimation and correction techniques (Anderson and Bezdek, 1984; Cazals and Pouget, 2005; Heikkila, 1998; Matas et al., 1995; Prasad, 2011; Prasad et al., 2011a; Worring and Smeulders, 1993; Zhong et al., 2009), partial retrieval of ellipses' parameters using some geometric properties of ellipses (Guil and Zapata, 1997; Ho and Chen, 1995; Yuen et al., 1989; Zhang and Liu, 2005) are usually added before the actual ellipse detection method. Further, most hybrid methods include some or other form of grouping mechanism to group the edges that possibly belong to the same ellipse (Chia et al., 2011; Hahn et al., 2008; Kawaguchi and Nagata, 1998b; Kim et al., 2002; Mai et al., 2008). Finally, there are some essential ellipse refinement and selection steps to deal with the outliers and reduce false positives (Basca et al., 2005; Cheng, 2006; Ji and Haralick, 2001; Princen et al., 1994; Qiao and Ong, 2007; Wang et al., 2007; Prasad et al., 2010e)).

\section{Least squares based high selectivity ellipse detection method}

The least squares methods currently in use are based on fundamental work by Rosin (Rosin, 1993a; Rosin, 1993b; Rosin, 1996a; Rosin, 1996b) and Fitzgibbon (Fitzgibbon et al., 1999), in which the algebraic equation of general conics is used for defining the minimization problem and additional numeric constraints are introduced in order to restrict the solutions to elliptic curves. The non-linear optimization problem is then solved for finding the parameters of the ellipses. These methods do not explicitly use the geometric properties of ellipse and as a consequence give high false positive and false negative rates.

We propose an elliptic geometry based least squares method that does not require constrained optimization and is highly selective of ellipses. Since it uses a set of 
unconventional variables which are related to the actual parameters of ellipses in a nonlinear manner. The constraints are directly incorporated in the definition of the new variables and the need of non-linear optimization is avoided. The main idea behind the proposed method is that since this method has to be applied on the digitized images (pixels), we can incorporate the effect of digitization in the development of least squares formulation. The main concept is that, rather than designing a least squares formulation using a general quadratic equation and satisfying certain constriants, we can use the geometric model of ellipse as the basic model and the distance of the pixels from the fitted ellipse as the criteria for designing the least squares formulation.

\subsection{Proposed method}

Consider the simplest form of ellipse, whose equation is as below:

$$
\frac{x^{2}}{a^{2}}+\frac{y^{2}}{b^{2}}=1
$$

For a point $P\left(x_{0}, y_{0}\right)$ on the ellipse, the equation of the tangent at the point is given as $y=-(b / a) \cot \theta_{0} x+b \csc \theta_{0}$, where we have used the parametric notation for the points on ellipses: $x_{0}=a \cos \theta_{0} ; y_{0}=b \sin \theta_{0}$. If the ellipse (1) has to be fitted on a sequence of pixels $P_{i}\left(x_{i}, y_{i}\right) ; x_{i}, y_{i} \in \mathbb{Z}$, we have to find $a$ and $b$ such that the distance of the pixels from the ellipse, or alternatively from the nearest tangents on the ellipse, is minimum. Thus, we want to minimize the residue:

$$
\min (\text { w.r.t } a, b):\left|y_{i}+\frac{b}{a} x_{i} \cot \theta_{0}-b \csc \theta_{0}\right|=\left|y_{i}+\frac{b^{2}}{a^{2}} \frac{x_{0}}{y_{0}} x_{i}-\frac{b^{2}}{y_{0}}\right|
$$

where $|\cdot|$ denotes the absolute value in the case of scalars and Euclidean norm in the case of vectors and the point $P\left(x_{0}, y_{0}\right)$ on the ellipse nearest to a pixel $P_{i}\left(x_{i}, y_{i}\right)$ satisfies $x^{\prime}=a \cos \theta_{0}+\Delta x, y^{\prime}=b \sin \theta_{0}+\Delta y$. Considering that the pixels in images are digitized form of the actual ellipse (rounding to the nearest integer), and assuming no other form of noise is present, it can be concluded that $|\Delta x|,|\Delta y| \leq 0.5$. In other words, the point $P\left(x_{0}, y_{0}\right)$ on the ellipse which is nearest to the pixel $P_{i}\left(x_{i}, y_{i}\right)$ is within one pixel region of $P_{i}\left(x_{i}, y_{i}\right)$. Thus, using $\Delta x, \Delta y \leq 0.5$, the intended maximum distance between $P_{i}\left(x_{i}, y_{i}\right)$ and $P\left(x_{0}, y_{0}\right)$ is $1 / \sqrt{2}$. Thus, we can write the upper limit of (2) as $\left|y_{i}+\left(b^{2} / a^{2}\right)\left(x_{0} / y_{0}\right) x_{i}-\left(b^{2} / y_{0}\right)\right| \leq 1 / \sqrt{2}$. The above indicates that there is a definite upper bound (which itself is very small) to the expression to be minimized. This indicates that the minimization problem (2) should be easily solvable. Since $P\left(x_{0}, y_{0}\right)$ is not known, but $|\Delta x|,|\Delta y| \leq 0.5$ are small, we can safely replace the values of $\left(x_{0}, y_{0}\right)$ with $\left(x_{i}, y_{i}\right)$ in (2) and rewrite (2) as follows:

$$
\min (\text { w.r.t } a, b):\left|y_{i}+\frac{b^{2}}{a^{2}} \frac{x_{i}^{2}}{y_{i}}-\frac{b^{2}}{y_{i}}\right|
$$

Thus, using the above minimization goal, we formulate a matrix equation as follows: 


$$
\left[\begin{array}{cc}
\vdots & \vdots \\
x_{i}^{2} / y_{i} & -1 / y_{i} \\
\vdots & \vdots
\end{array}\right]\left[\begin{array}{c}
b^{2} / a^{2} \\
b^{2}
\end{array}\right]=\left[\begin{array}{c}
\vdots \\
-y_{i} \\
\vdots
\end{array}\right]
$$

However, for numerical stability, (4) can be modified as:

$$
\left[\begin{array}{cc}
\vdots & \vdots \\
x_{i}^{2} & -1 \\
\vdots & \vdots
\end{array}\right]\left[\begin{array}{c}
b^{2} / a^{2} \\
b^{2}
\end{array}\right]=\left[\begin{array}{c}
\vdots \\
-y_{i}^{2} \\
\vdots
\end{array}\right]
$$

The above model is used to compute values of $b^{2}$ and $b^{2} / a^{2}$ using least squares inversion (Weisstein), which can be used subsequently to calculate the values of $a$ and $b$. Following the same logic, we can formulate the least squares problem for a general ellipse, with centre at $O(\tilde{x}, \tilde{y})$, semi-major and semi-minor axes $a$ and $b$, and the angle of orientation $\alpha$. For brevity, we present the model without incorporating the actual details of derivation:

$$
\left[\begin{array}{ccccc}
\vdots & \vdots & \vdots & \vdots & \vdots \\
x_{i}^{2} & 2 x_{i} y_{i} & -2 x_{i} & -2 y_{i} & -1 \\
\vdots & \vdots & \vdots & \vdots & \vdots
\end{array}\right]\left[\begin{array}{lllll}
\phi_{1} & \phi_{2} & \phi_{3} & \phi_{4} & \phi_{5}
\end{array}\right]^{\mathrm{T}}=\left[\begin{array}{c}
\vdots \\
-y_{i}^{2} \\
\vdots
\end{array}\right]
$$

and the parameters of the ellipse can be found using:

$$
\begin{gathered}
\tilde{x}=\left(\phi_{3}-\phi_{4} \phi_{2}\right) /\left(\phi_{1}-\phi_{2}^{2}\right) ; \tilde{y}=\left(\phi_{1} \phi_{4}-\phi_{3} \phi_{2}\right) /\left(\phi_{1}-\phi_{2}{ }^{2}\right) ; \alpha=0.5 \tan ^{-1}\left(2 \phi_{2} /\left(\phi_{1}-1\right)\right) \\
a=\sqrt{2\left(\phi_{5}+\tilde{y}^{2}+\tilde{x}^{2} \phi_{1}+2 \phi_{2}\right) /\left(\left(1+\phi_{1}\right)-\sqrt{\left(1-\phi_{1}\right)^{2}+4 \phi_{2}^{2}}\right)} \\
b=\sqrt{2\left(\phi_{5}+\tilde{y}^{2}+\tilde{x}^{2} \phi_{1}+2 \phi_{2}\right) /\left(\left(1+\phi_{1}\right)+\sqrt{\left(1-\phi_{1}\right)^{2}+4 \phi_{2}^{2}}\right)}
\end{gathered}
$$

\subsection{Numerical examples}

In this section, we consider various numerical examples and compare the performance of the proposed method with Fitzgibbon's method (Fitzgibbon et al., 1999). We consider three main categories of curves: elliptic, non-elliptic conical, and non-conical curves. Using the results, we demonstrate that the proposed method is more robust and generates less false positives than Fitzgibbon (Fitzgibbon et al., 1999).

\subsubsection{Elliptic curves}

For elliptic curves, we consider three experiments. The experiments are performed on a family of digital ellipses, whose minimum semi-minor distance is 10 pixels, and maximum semi-major distance is 2000 pixels. Thus, the eccentricities of the ellipses may vary between 0 and 0.999987 . The centre of the ellipses lie within a square region of size 2000 pixels and centred at the origin. The angle of orientation lies in the range $\left[-90^{\circ}, 90^{\circ}\right]$. For comparing the actual ellipses with the detected ellipses, we use the following measures: 


$$
\begin{gathered}
\delta \tilde{x}=\left|\tilde{x}-\tilde{x}_{e s t}\right| ; \delta \tilde{y}=\left|\tilde{y}-\tilde{y}_{e s t}\right| ; \delta a=\left|a-a_{e s t}\right| / a ; \quad \delta b=\left|b-b_{e s t}\right| / b ; \\
\delta \alpha=\left|\alpha-\alpha_{e s t}\right| \sqrt{1-b^{2} / a^{2}} \text { (in degrees) }
\end{gathered}
$$

where the subscript 'est' is used to denote the values estimated by the ellipse detection method.

In practice, the complete elliptic curve is not available due to occlusion or lighting conditions. Thus, it is important that an ellipse detection method is capable of detecting ellipses from partial curves as well. This is the motivation of this example. We consider the curves from $\left[\left(\theta_{0}+\alpha\right),\left(\theta_{0}+\alpha+\Delta \theta\right)\right]$, where we vary $\Delta \theta$ from $90^{\circ}$ to $360^{\circ}$ at a step of $10^{\circ}$, thus the experiment contains 36 cases. For each case, we generate 10,000 digital elliptic curves. For each of the curve, the parameters $\tilde{x}, \tilde{y}, a, b, \alpha$, and $\theta_{0}$ are generated randomly. The range for $\theta_{0}$ is $\left[0,360^{\circ}\right]$.

The measures in (8) are computed for each curve and then averaged for all the 10,000 curves corresponding to a particular value of $\Delta \theta$. The residues and the measures (8) are presented in Fig. 4.

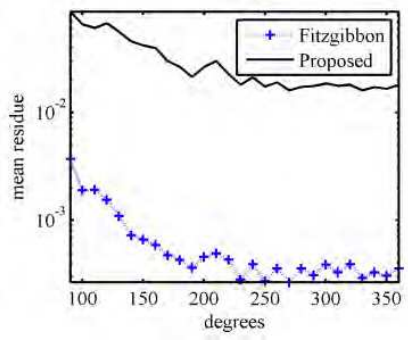

(a) residue vs. $\Delta \theta$

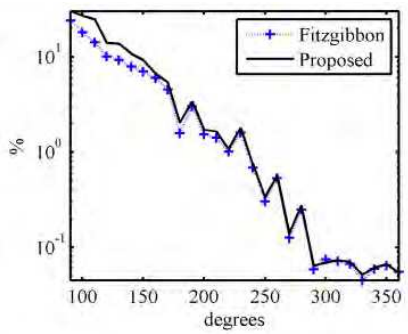

(d) $\delta a$ vs. $\Delta \theta$

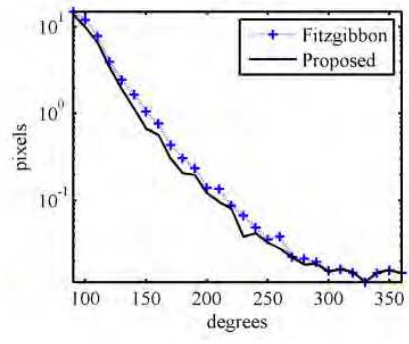

(b) $\delta \tilde{x}$ vs. $\Delta \theta$

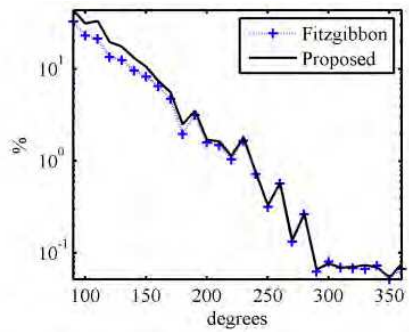

(e) $\delta b$ vs. $\Delta \theta$

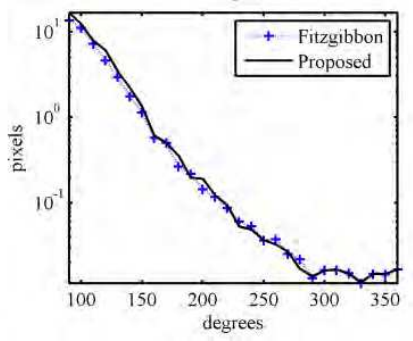

(c) $\delta \tilde{y}$ vs. $\Delta \theta$

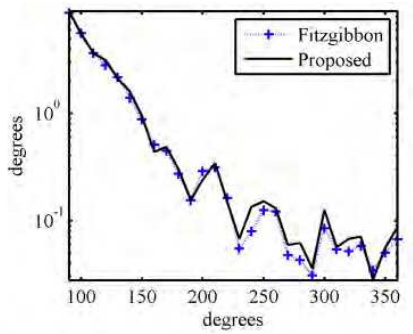

(f) $\delta \alpha$ vs. $\Delta \theta$

Fig. 4. Residue and error measures for experiment in section 3.2.1.

The errors are very small when we have the entire curve available, i.e., $\Delta \theta=360^{\circ}$. However, even for other values $\Delta \theta>90^{\circ}$, the errors are in practically useful range. We note that Fitzgibbon (Fitzgibbon et al., 1999) generated invalid conics for about $56 \%$ of the all the curves, thus indicating a false negative rate of $56 \%$. We also note that the smaller the curve, the higher is the error for all the parameters, which is expected, since lesser and lesser 
curvature is available. Further, $\delta a$ and $\delta b$ are very large for small curves, indicating that larger curve is essential for detecting the ellipses accurately. It is seen that though Fitzgibbon (Fitzgibbon et al., 1999) has lower residue, the proposed method has lower or comparable error for almost all the parameters.

\subsubsection{Non-elliptic conical curves}

We intend to test if Fitzgibbon (Fitzgibbon et al., 1999) and the proposed method generate false positives, i.e., detect non-elliptic curves as ellipses. For this, we consider a family of conics given by:

$$
x=l \cos \theta /(1-e \cos \theta) ; y=l \sin \theta /(1-e \cos \theta)
$$

where $l \in[20,200]$ and eccentricity $e \in[1,2]$. We generate portions of this curve corresponding to $\theta \in[180-\Delta \theta / 2,180+\Delta \theta / 2]$, where $\Delta \theta$ is stepped from $45^{\circ}$ to $180^{\circ}$ in steps of $5^{\circ}$. As before, for each value of $\Delta \theta, 10,000$ random curves using (9) are generated. The family of curves corresponding to $\Delta \theta=180^{\circ}$ is shown in Fig. 5(a). It is seen that though the residue is small for the proposed method (Fig. 5(b)), the proposed method identifies all the curves as non-elliptic (i.e., generates imaginary value for at least one of the parameters $a$ and $b$ of the fitted ellipse). On the other hand, Fitzgibbon (Fitzgibbon et al., 1999) fits some real valued ellipses on the most non-elliptic curves as well (see Fig. 5(c)). When the curves are digitized, though the performance of the proposed method gets poor, for small values of $\Delta \theta$, in general, it still outperforms Fitzgibbon (Fitzgibbon et al., 1999) in its selectivity for the elliptic curves (see Fig. 5(d)).

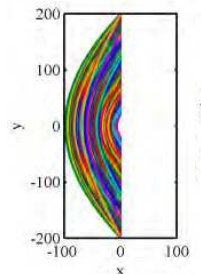

(a) family of conics

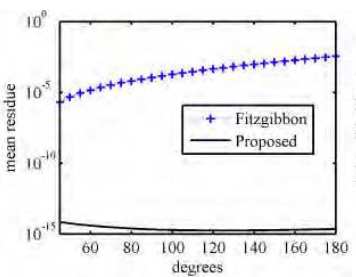

(b) residue vs. $\Delta \theta$

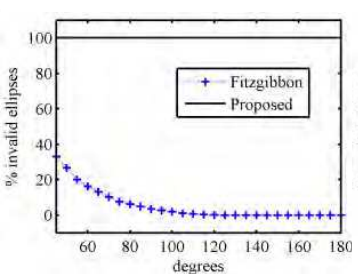

(c) \% of true negative detections

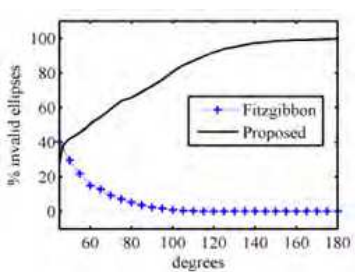

(d) \% of true negative detection (in the presence of digitization)

Fig. 5. Residue and percentage of invalid ellipses for experiment 3.2.2.

\subsubsection{Non-conical fourth order curves}

Now, we consider a family of curves given by the equation:

$$
\frac{x^{4}}{a^{4}}+\frac{y^{4}}{b^{4}}=1
$$

where $a, b \in[20,200], x=a \sqrt{\cos \theta}, x=b \sqrt{\sin \theta}$, and $\theta \in[0, \Delta \theta]$. For this family, we step $\Delta \theta$ from $45^{\circ}$ to $90^{\circ}$ at steps of $5^{\circ}$. As before, for each value of $\Delta \theta$, we generate 10,000 
randomly chosen curves. The family of curves for $\Delta \theta=90^{\circ}$ is plotted in Fig. 6(a). In the absence of digitization, Fitzgibbon (Fitzgibbon et al., 1999) is not at all selective about elliptic curves, while the proposed method rejects all the curves as non-elliptic (see Fig. 6(b)). In the presence of digitization, though the selectivity of the proposed method decreases for small values of $\Delta \theta$, Fitzgibbon (Fitzgibbon et al., 1999) continues to perform poorly (see Fig. 6(c)).

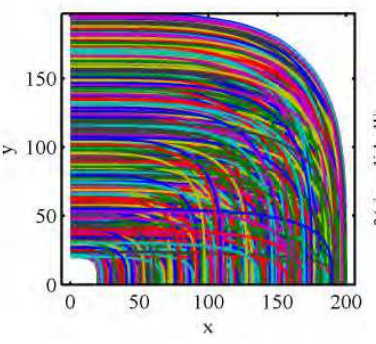

(a) family of curves

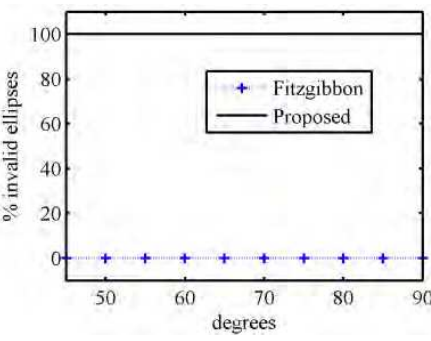

(b) \% of true negative detections

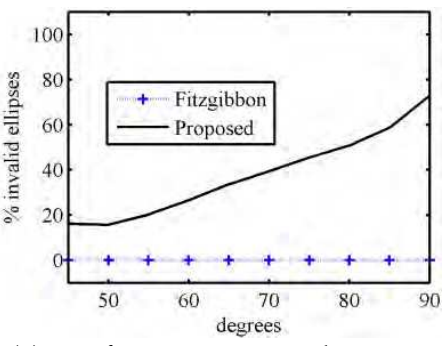

(c) \% of true negative detection (in the presence of digitization)

Fig. 6. Percentage of invalid ellipses for experiment 3.2.3.

\section{Hybrid ellipse detection method for real images}

We propose a hybrid ellipse detection method for real images. The proposed method consists of three major stages, viz., edge preprocessing, ellipse detection, and salient ellipse selection. Various steps in these methods address to various problems in the problem of ellipse detection. We succinctly highlight the steps which serve an important purpose in the algorithm in the following list:

- The dominant point detection (step 3 of section 4.1) is helpful in reducing the effect of digitization and corruption in the quality of edges due to noise.

- $\quad$ The curvature correction (step 4 of section 4.1) is useful for dealing with the corruption in the quality of image due to light and shadow conditions. Specifically, it is useful for dealing with the merging of edges of two objects, with one or both possibly being elliptic.

- The determination of the search region (step 1 of section 4.2) is useful in making the method more selective of ellipses by targeting two problems, looking for the presence of incomplete ellipses and simultaneously reducing the possibility of obvious outliers (which will definitely not present in the search region of an edge).

- $\quad$ The associated convexity (step 2 of section 4.2) further increases the method's selectivity for ellipses by weeding out other edges that may be outliers.

- The geometric center finding (step 3 of section 4.2) is the core step that enables the grouping of edges possibly belonging to the same ellipse. In conjunction with steps 1 and 2 of section 4.2, this step enables the grouping of the edges that possibly belong to the same ellipse with much greater reliability than most contemporary methods.

- The relationship score (step 4 of section 4.2) is a technique of quantifying the reliability of relevance of an edge in the candidate ellipse. This technique enables the ellipse detection in the next step with very good reliability because the next step can further remove some edges with lower relationship score (thus identifying them as outliers). 
- $\quad$ Detecting similar ellipses (step 1 of section 4.3) is a way of reducing multiple detections for one elliptic object in the image. This step chooses the best representative ellipse for an object, thus improving the selectivity of the method.

- The saliency parameters (steps $2-5$ of sections 4.3) quantify the quality of detected ellipses (precision as well as reliability) which are used in step 6 of section 4.3 for further reducing the false positives. Step 6 of section 4.3 is a technique of nonheuristically choosing the detected ellipses with above average quality.

\subsection{Edge preprocessing}

The various steps of this stage are listed below:

1. Extraction of edge map: The real image is converted to the gray image and Canny edge detector is used to obtain the edge map.

2. Obtaining edges: Then, sequences of continuous edge pixels are identified, and called edges. The edges begin and end with either 1-connected or $>2$ connected edge pixels. There are many approaches in vogue for implementing this step. We use the codes by (Kovesi) in our method. But, other methods may be used as well.

3. Dominant point detection (polygonal approximation of edges): After this, we approximate the edges with a polygonal approximation (or dominant point detection method). We note that we have used a recently proposed parameter independent line fitting method which provides robust approximation for edges of any length and curvature and noise (Prasad et al., 2011b). In general, if other polygonal approximation methods are used, the performance depends upon the control parameters very strongly. Also, if these methods are used, we note that one value of control parameter may be suitable for one image, but may result in poor performance for another image. Such issues are absent in (Prasad et al., 2011b) and it provides good performance for all the images used for testing the performance of the algorithm.

4. Curvature correction: The sequence of dominant points obtained for an edge is used to perform curvature correction. For this step, it is useful to define the sequence of chords of the polygonal approximation of an edge $e$. Suppose $\left\{l_{1}, l_{2}, \ldots, l_{N}\right\}$ is the sequence of chords formed by the dominants point of the edge $e$. Let the angles between all the pairs of consecutive line segments be denoted as $\left\{\theta_{1}, \theta_{2}, \ldots, \theta_{N-1}\right\}$, where $\theta_{i} \in[-\pi, \pi]$ is the anticlockwise angle from $l_{i+1}$ to $l_{i}$ (see Fig. 7(a) for illustration).

a. Removal of sharp turns: In the sequence of the angles, $\left\{\theta_{1}, \theta_{2}, \ldots, \theta_{N-1}\right\}$, if any angle $\theta_{i}$ is large, i.e., equal to or greater than a chosen threshold $\theta_{0}$ (say $\pi / 2$, empirically determined), then the change in the curvature of the edge at such points $P_{i+1}$ (the intersection point of line segments $l_{i}$ and $l_{i+1}$ ) is considered to be large (or sharp), and the edge is split at $P_{i+1}$ to ensure smooth curvature.

b. Removal of inflexion points: From the above definition of the angles $\left\{\theta_{1}, \theta_{2}, \ldots, \theta_{N-1}\right\}$, the change in direction of curvature occurs in the change of the sign of the angles (negative or positive). Thus, we can create a Boolean sequence $\left\{b_{1}, b_{2}, \ldots, b_{N-1}\right\}$, where $b_{i}$ is ' 0 ' if the sign of $\theta_{i}$ and $\theta_{1}$ is the same. This Boolean sequence can be used to identify the inflexion points and decide the exact places where the edge contour should be split. The three possibilities of occurrence of inflexion points are shown in Fig. 7(b). The points where the edge needs to be split are also shown in the same figure. 


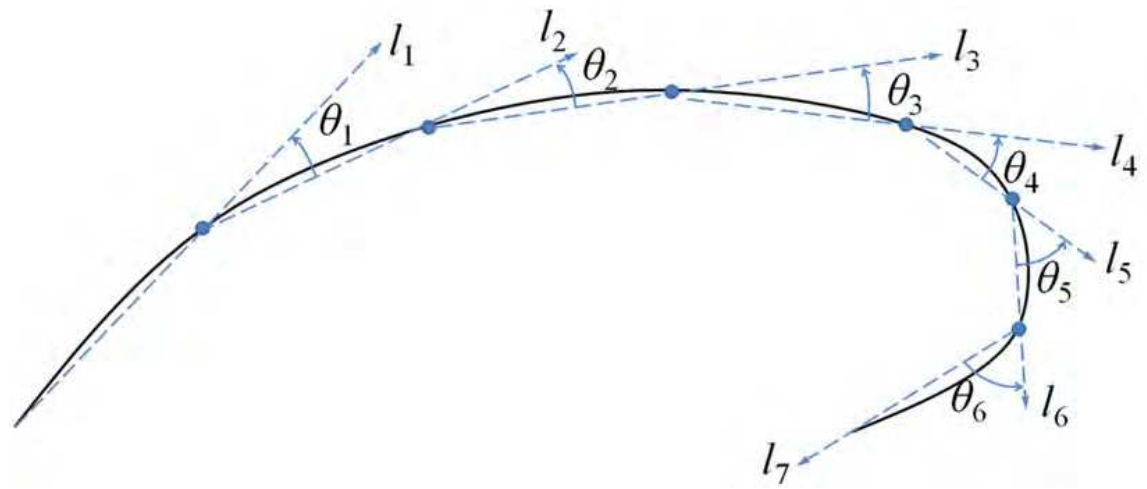

(a) Illustration of the chords formed by the dominant points (polygonal approximation) of an edge
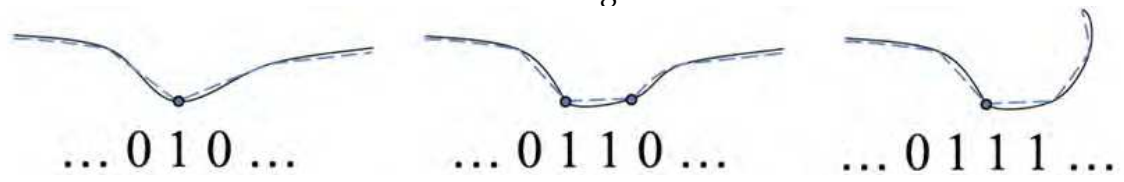

(b) Illustration of varieties of inflexion points and the dominant points to be deleted

Fig. 7. Illustrations for the curvature correction techniques used in step 4 of section 4.1.

\subsection{Ellipse detection}

The various steps of this stage are discussed below:

1. Determining search region of an edge: We define the search region $R$ as following. For a given edge $e$, let the tangents to the edge at its end points $P_{1}$ and $P_{2}$ be denoted by $l_{1}$ and $l_{2}$, and the line segment connecting the end points $P_{1}$ and $P_{2}$ be denoted by $l_{3}$. The two tangents $l_{1}$ and $l_{2}$ and the line $l_{3}$ divide the image into two regions, $R_{1}$ and $R_{2}$, as shown in Fig. 8(a). Then the search region $R$ is the region that does not contain $P_{\text {mid }}$, where $P_{\text {mid }}$ is the middle pixel of the edge $e$. Mathematically, the search region $R$ is defined as follows:

$$
R= \begin{cases}R_{2} & P_{\text {mid }} \notin R_{2} \\ R_{1} & \text { otherwise }\end{cases}
$$

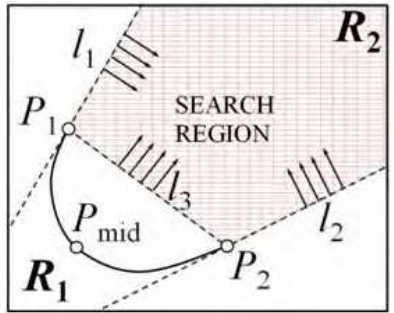

(a) Search region

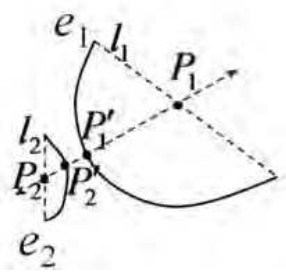

(b) demonstration of associated convexity

Fig. 8. Illustrations of search region and associated convexity (steps 1 and 2 of section 4.2.) 
2. Computing associated convexity: Let us consider the line segments $l_{1}$ and $l_{2}$ formed by joining the end points of $e_{1}$ and $e_{2}$, respectively, as shown in Fig. 8. Let $P_{1}$ and $P_{2}$ be the midpoints of the line segments $l_{1}$ and $l_{2}$. Let $l_{3}$ be a line passing through $P_{1}$ and $P_{2}$, such that it intersects the edges $e_{1}$ and $e_{2}$ at $P_{1}^{\prime}$ and $P_{2}^{\prime}$ respectively. The pair of edges $e_{1}$ and $e_{2}$ are suitable for grouping if and only if:

$$
P_{1}^{\prime} P_{2}^{\prime} \approx P_{1} P_{1}^{\prime}+P_{1} P_{2}+P_{2} P_{2}^{\prime}
$$

3. Detection of the geometric center of the ellipse corresponding to an edge: In this step, we use the method proposed in (Yuen et al., 1989) for estimating the geometric center of the ellipse to which the edge may belong. This method uses the tangents estimated at three points on the edge. It was shown in (Prasad and Leung, 2010b) that Yuen's method is sensitive to the error in tangent estimation. Thus, we use a recently proposed upperbounded tangent estimator for digital curves (Prasad et al., 2011a) using $R=4$, which gives better performance than other contemporary tangent estimation methods. We split an edge into three sub-edges and choose points randomly from each sub-edge to form several (upto 200) sets of three points. Then for each set, a geometric center is found.

4. Determination of relationship score: The image space is divided into equal square bins where the size of one bin is given using the Rayleigh distribution presented in (Prasad and Leung, 2010b). For an edge and a bin, the relationship score proposed in (Prasad and Leung, 2010a) is given by:

$$
r_{e}^{b}=S_{e}^{b} r_{1} r_{2}
$$

where $r_{1}=\left(\frac{S_{e}^{b}}{S_{e}}\right) \exp \left(\frac{S_{e}^{b}}{S_{e}}-1\right), r_{2}=\left(\frac{S_{e}}{S}\right) \exp \left(2\left(\frac{S_{e}}{S}-1\right)\right), S=200$ is the total number of sets of three pixels, $S_{e}$ is the number of sets for which no geometric exception occurred, and $S_{e}^{b}$ is the number of sets that generated geometric centers inside the square bin $b$. More details can be found in (Prasad and Leung, 2010a).

5. Grouping of edges and ellipse detection: All the edges having a common bin $b$ may initially be considered as a group. However, every edge in the group should also fall in the search region of every other edge and satisfy the condition of associated convexity. The edges that do not fulfill these conditions are removed from the group. The edges in a group are ranked in the descending order of their bin-edge relationship scores $r_{e}^{b}$. The edge pixels of the edges in a group are appended and least squares fitting technique (Fitzgibbon et al., 1999) 2 is used on the group to find all the parameters of the ellipse. Now, the quality of the group is evaluated using the two criteria listed below:

- Criterion $1(\mathrm{C} 1)$ : Error of least squares fitting $\leq \varepsilon_{l s}$, a chosen threshold error value.

- Criterion 2 (C2): The bin $b$ of the group is inside the detected elliptic hypothesis.

the threshold used for $\mathrm{C} 1$ is $\varepsilon_{l s}=0.01$. If both $\mathrm{C} 1$ and $\mathrm{C} 2$ are satisfied, then the parameters of the ellipses computed using the least squares fitting are passed to the next stage. If

\footnotetext{
${ }^{2}$ We recommend that the least squares method presented in section 3 is used. However, the results for section 4 using the method in section 3 were not available at the time of writing this chapter.
} 
anyone of the two criterions is not fulfilled, the weakest edge (with the lowest relationship score $r_{e}^{b}$ ) is removed from the group and the above process is repeated till either the above criteria are satisfied or the group becomes empty.

\subsection{Salient ellipse selection}

The various steps of this stage are discussed below:

1. Detecting similar ellipses: For two ellipses $E_{1}$ and $E_{2}$, we obtain the Boolean matrices $I_{1}$ and $I_{2}$ of the same size as the actual image, such that the pixels outside the ellipse are assigned Boolean ' 0 ' amd the remaining are assigned Boolean ' 1 '. Then, the similarity measure $D$ is computed using:

$$
D=1-\frac{\operatorname{count}\left(X O R\left(I_{1}, I_{2}\right)\right)}{\operatorname{count}\left(O R\left(I_{1}, I_{2}\right)\right)}
$$

where $\operatorname{count}(A)$ gives the number of Boolean ' 1 ' elements in the matrix $A$. For a given ellipse, all the ellipses that have overlap ratio $D>0.9$ are clustered together. Among the ellipses in a cluster, the choice of representative candidate is done by choosing the ellipse that was formed by maximum amount of data. Thus, we have used percentage circumference of an ellipse (15) for choosing the representative.

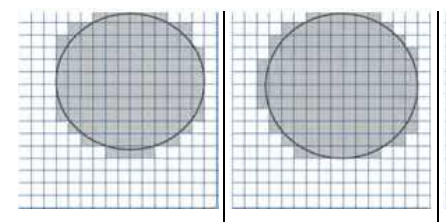

(i) ellipse $E_{1}$ and (ii) ellipse $E_{2}$ and Boolean matrix $I_{1}$

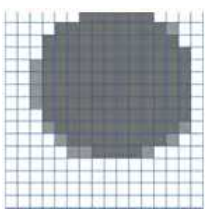

(iii) light gary pixels denote $\operatorname{XOR}\left(I_{1}, I_{2}\right)$

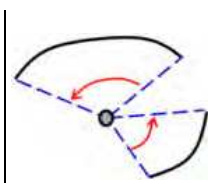

(i) angular circumference ratio

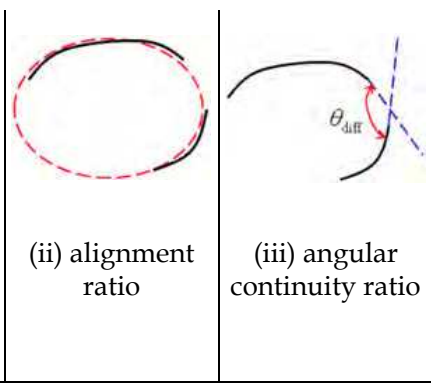

(b) Illustration of the saliency measures

(a) Illustration of the similarity measure (D)

Fig. 9. The illustration of the concept of similarity measures and the saliency measures

2. Computing circumference ratio: Suppose an ellipse $E$ was fitted to a group $G$, then we define circumference ratio $c(E, G)$ as below:

$$
c(E, G)=\sum_{\forall e \in G} \alpha(E, e) / 2 \pi
$$

where $\alpha(E, e)$ is the angle subtended by the ends of the edge $e$ at the centre of the ellipse $E$. A higher value of $c(E, G)$ implies a larger support of $E$ on $G$.

3. Computing alignment ratio: We consider all the pixels the pixels $\left\{P_{i} ; i=1\right.$ to $\left.N_{G}\right\}$ in the group of edges that generated an elliptic hypothesis, and compute their Euclidean distance $d_{i}$ from the elliptic hypothesis. This is used to compute a function $s\left(E, P_{i}\right)$ as follows: 


$$
s\left(E, P_{i}\right)=\left\{\begin{array}{lc}
1 & \text { if } d_{i}<d_{0} \\
0 & \text { otherwise }
\end{array}\right.
$$

The value of threshold chosen here is $d_{0}=2$. Then the alignment ratio is calculated as:

$$
a(E, G)=\sum_{i=1}^{N_{G}} s\left(E, P_{i}\right) / N_{G}
$$

4. Computing angular continuity ratio: The angular continuity ratio is computed as:

$$
\phi(E, G)=\left\{\begin{array}{cc}
1 & \text { if } N=1 \\
\frac{1}{N-1} \sum_{i=1}^{N-1} \frac{\theta_{\text {diff }}\left(e_{i}, e_{i+1}\right)}{\pi} & \text { if } N>1
\end{array}\right.
$$

where $N$ is the number of edge curves in the group $G$.

5. Computing net saliency score: The net saliency score is computed using circumference ratio, alignment ratio, and angular continuity ratio, as follows:

$$
\sigma_{\mathrm{add}}(E, G)=\frac{a(E, G)+c(E, G)+\phi(E, G)}{3} .
$$

6. Final selection of ellipses: In order to make the selection of the elliptic hypotheses nonheuristic, the decision of selecting the elliptic hypothesis $E$ is made using the expression below:

$$
\operatorname{AND}\left(\begin{array}{c}
a(E, G) \geq \operatorname{avg}\{a(E, G)\}, \\
c(E, G) \geq \operatorname{avg}\{c(E, G)\}, \\
\phi(E, G) \geq \operatorname{avg}\{\phi(E, G)\}, \\
\sigma_{\text {add }}(E, G) \geq \operatorname{avg}\left\{\sigma_{\text {add }}(E, G)\right\}
\end{array}\right)
$$

Here, $\operatorname{avg}\{a(E, G)\}$ is the average value of the alignment ratios calculated for all the elliptic hypotheses remaining after the similar ellipses identification. The same applies for the other expressions in (20).

\section{Experimental results for section 4}

\subsection{Synthetic dataset: Overlapping and occluded ellipses}

To generate the synthetic images, we consider an image size of $300 \times 300$ and generate $\alpha \in\{4,8,12,16,20,24\}$ ellipses randomly within the region of image. The parameters of the ellipses are generated randomly: center points of the ellipses are arbitrarily located within the image, lengths of semi-major and semi-minor axes are assigned values randomly from the range $[10,300 / \sqrt{2}]$, and the orientations of the ellipses are also chosen randomly. The only constraint applied is that each ellipse must be completely contained in the image and overlap with at least one ellipse. For each value of $\alpha$, we generate 100 images containing 
occluded ellipses and 100 other images containing overlapping ellipses. In the occluded ellipses, the edges of the overlapped regions are not available, while in the overlapping images all the edge contours of the ellipses are available. Thus, in total there are 600 images with occluded ellipses and 600 images with overlapping ellipses.

The true positive elliptic hypotheses are identified as the elliptic hypotheses that have high overlap with the ellipses in the ground truth. We use precision, recall, and F-measure (Baeza-Yates and Ribeiro-Neto, 1999) for measuring the performance of the proposed method. The performance of method is compared with the results of Mai (Mai et al., 2008), Kim (Kim et al., 2002), simplified Hough transform (McLaughlin, 1998), and randomized Hough transform (McLaughlin, 1998). The comparative results are presented in Table 1 for synthetic images with occluded ellipses and in Table 2 for synthetic images with overlapping ellipses.

The proposed method gives the best performance among all the methods considered for both occluded and overlapping ellipses. Some example images are also provided in Fig. 10.

\subsection{Synthetic dataset: Overlapping and occluded ellipses}

Now, we compare the performance of various methods and the proposed method (scheme 3) for the real image dataset. The average value of the performance metrics for the 400 real

\begin{tabular}{|c|c|c|c|c|}
\hline \multicolumn{5}{|c|}{ Recall } \\
\hline$\alpha$ & Hybrid method (section 4) & Chia & Mai & Kim \\
\hline 4 & 0.99 & 0.90 & 0.39 & 0.48 \\
\hline 8 & 0.98 & 0.80 & 0.20 & 0.20 \\
\hline 12 & 0.95 & 0.70 & 0.13 & 0.09 \\
\hline 16 & 0.92 & 0.65 & 0.05 & 0.05 \\
\hline 20 & 0.90 & 0.62 & 0.04 & 0.04 \\
\hline 24 & 0.86 & 0.55 & 0.01 & 0.02 \\
\hline \multicolumn{5}{|c|}{ Precision } \\
\hline 4 & 0.93 & 0.9 & 0.62 & 0.75 \\
\hline 8 & 0.91 & 0.82 & 0.42 & 0.59 \\
\hline 12 & 0.89 & 0.73 & 0.34 & 0.36 \\
\hline 16 & 0.87 & 0.70 & 0.18 & 0.30 \\
\hline 20 & 0.85 & 0.68 & 0.12 & 0.20 \\
\hline 24 & 0.82 & 0.60 & 0.04 & 0.18 \\
\hline \multicolumn{5}{|c|}{ F-measure } \\
\hline 4 & 0.96 & 0.90 & 0.48 & 0.58 \\
\hline 8 & 0.94 & 0.80 & 0.28 & 0.3 \\
\hline 12 & 0.91 & 0.71 & 0.19 & 0.15 \\
\hline 16 & 0.90 & 0.69 & 0.09 & 0.10 \\
\hline 20 & 0.87 & 0.65 & 0.05 & 0.06 \\
\hline 24 & 0.84 & 0.58 & 0.01 & 0.05 \\
\hline
\end{tabular}

Table 1. Result of the hybrid ellipse detection method for synthetic images with occluded ellipses. 
images is shown in Table 3. The total time taken by each method for the complete dataset is also shown. In terms of the performance, the proposed method not only outperforms the other methods, it also shows practically acceptable level of performance.

Though the time taken by Mai (Mai et al., 2008) is small, the proposed method takes lesser time than the remaining methods. Further, the superior performance of the proposed method as compared to Mai (Mai et al., 2008) clearly outweighs the longer time taken by the proposed method. The proposed method is easily parallelizable and can give real time performance when optimized for specific applications.

We also give some examples of real images and our results in Fig. 11 to Fig. 14.

\begin{tabular}{|c|c|c|c|c|}
\hline \multicolumn{5}{|c|}{ Recall } \\
\hline$\alpha$ & Hybrid method (section 4) & Chia & Mai & Kim \\
\hline 4 & 1.00 & 1.00 & 0.78 & 0.80 \\
\hline 8 & 0.99 & 0.96 & 0.62 & 0.55 \\
\hline 12 & 0.98 & 0.96 & 0.53 & 0.35 \\
\hline 16 & 0.95 & 0.87 & 0.45 & 0.20 \\
\hline 20 & 0.91 & 0.79 & 0.35 & 0.10 \\
\hline 24 & 0.89 & 0.69 & 0.32 & 0.09 \\
\hline \multicolumn{5}{|c|}{ Precision } \\
\hline 4 & 1.00 & 1.00 & 0.81 & 0.90 \\
\hline 8 & 0.98 & 0.96 & 0.70 & 0.81 \\
\hline 12 & 0.95 & 0.96 & 0.65 & 0.75 \\
\hline 16 & 0.94 & 0.91 & 0.58 & 0.64 \\
\hline 20 & 0.92 & 0.89 & 0.48 & 0.44 \\
\hline 24 & 0.90 & 0.82 & 0.46 & 0.44 \\
\hline \multicolumn{5}{|c|}{ F-measure } \\
\hline 4 & 1.00 & 1.00 & 0.79 & 0.85 \\
\hline 8 & 0.98 & 0.96 & 0.66 & 0.66 \\
\hline 12 & 0.97 & 0.96 & 0.48 & 0.59 \\
\hline 16 & 0.94 & 0.90 & 0.30 & 0.50 \\
\hline 20 & 0.91 & 0.82 & 0.15 & 0.41 \\
\hline 24 & 0.89 & 0.75 & 0.14 & 0.39 \\
\hline
\end{tabular}

Table 2. Result of the hybrid ellipse detection method for synthetic images with overlapping ellipses. 




Fig. 10. Example synthetic image and ellipses detected on the images. 


\begin{tabular}{lccc}
\hline & Hybrid method (section 4) & Mai & Kim \\
\hline Average Precision & 0.8748 & 0.2862 & 0.1831 \\
Average Recall & 0.7162 & 0.1632 & 0.1493 \\
Average F-measure & 0.7548 & 0.1831 & 0.1591 \\
$\begin{array}{l}\text { Average time taken } \\
\text { (seconds) }\end{array}$ & 38.68 & 11.41 & 60.87 \\
\hline
\end{tabular}

Table 3. Performance metrics for the proposed method (Section 4), Mai (Mai et al., 2008), and Kim (Kim et al., 2002) for real dataset (400 real images (Griffin et al.)).
(a) Original Image
(b) Canny edge map
(c) Extracted edges
(d) Detected ellipses
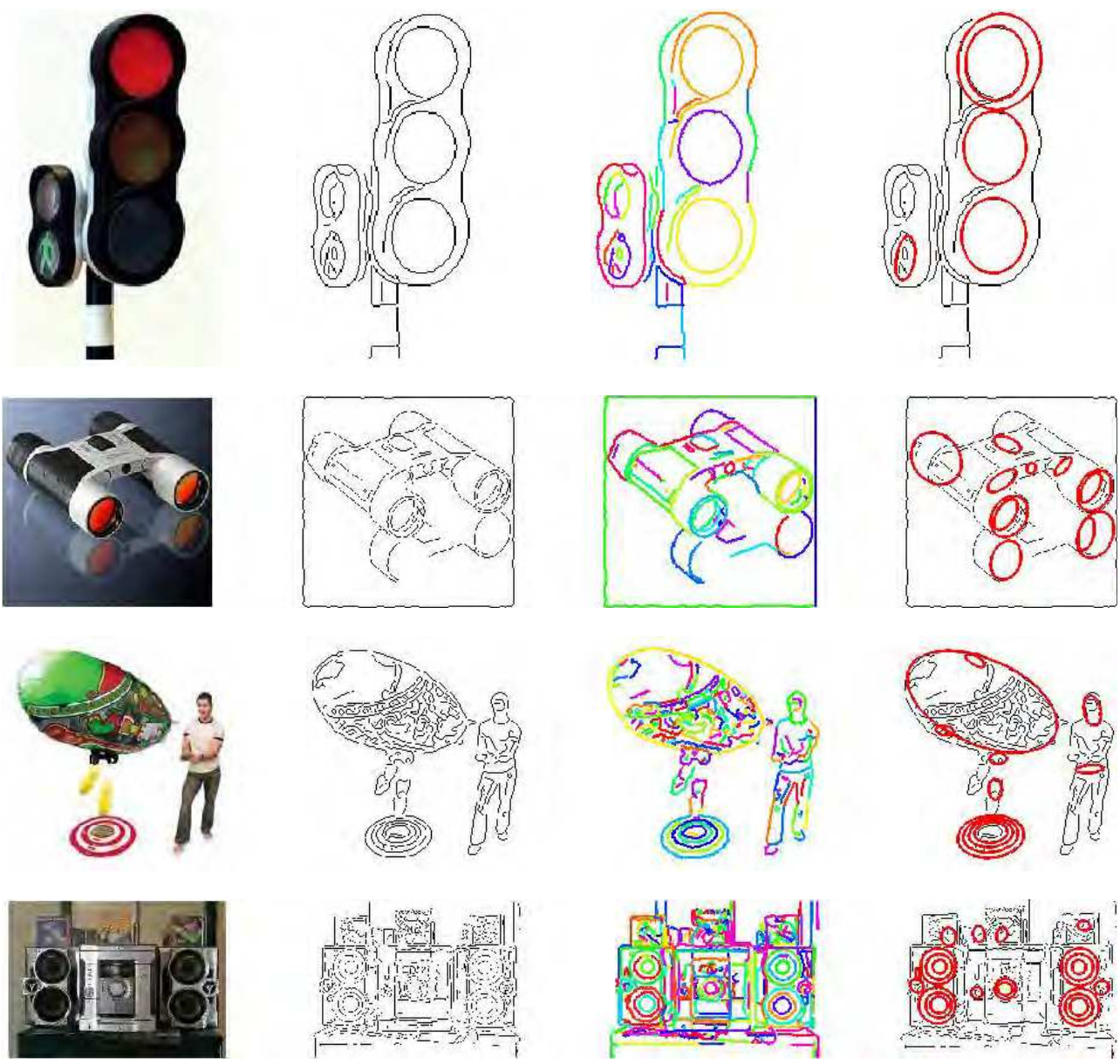

Fig. 11. Examples of real images and ellipse detection using the proposed method. 
(a) Original Image
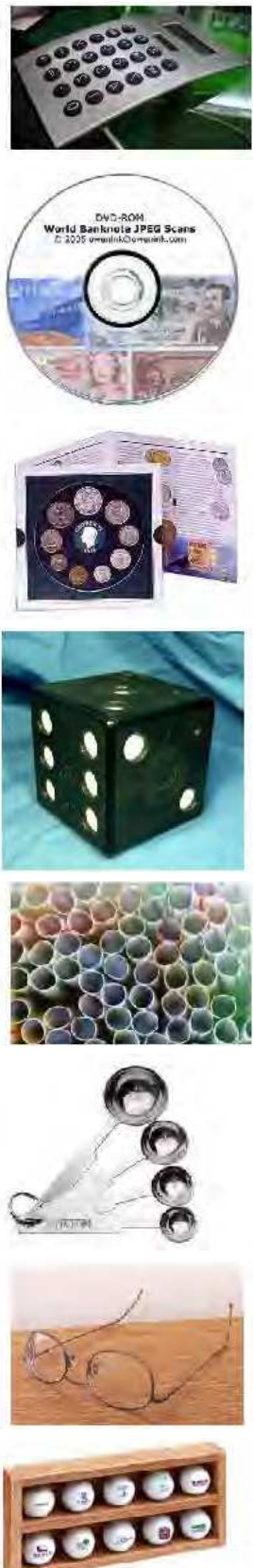

(b) Canny edge map
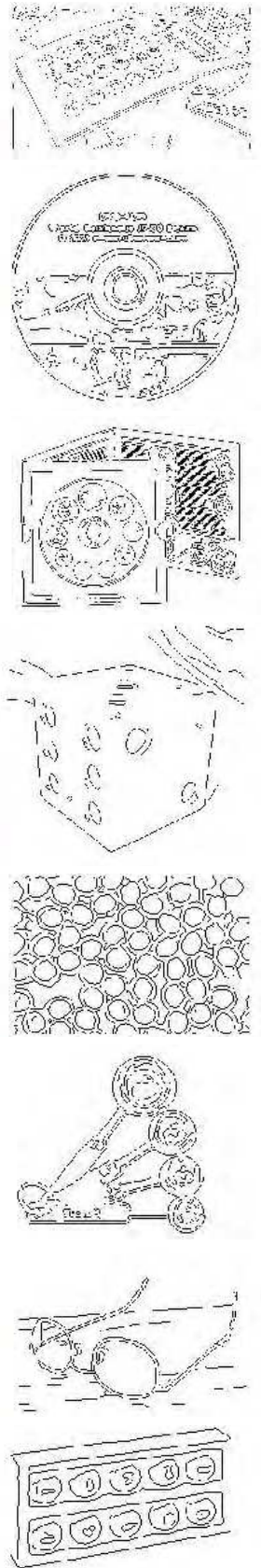

(c) Extracted edges
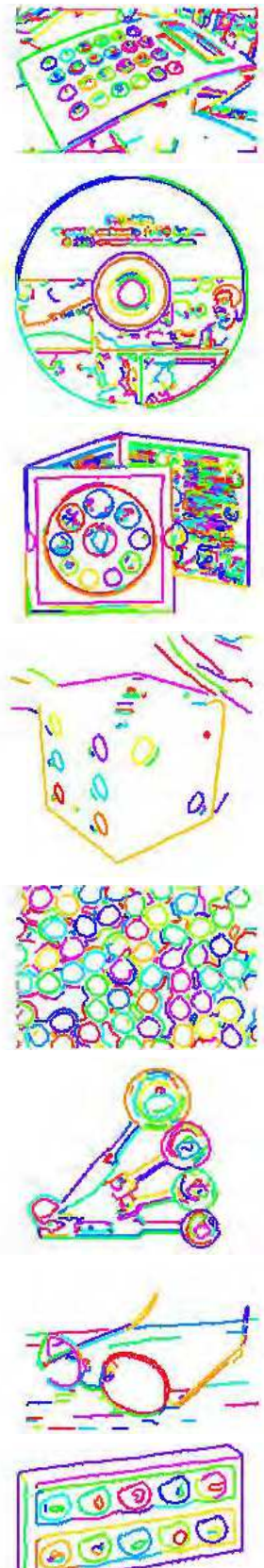

(d) Detected ellipses


Fig. 12. Examples of real images and ellipse detection using the proposed method (continued). 
(a) Original Image
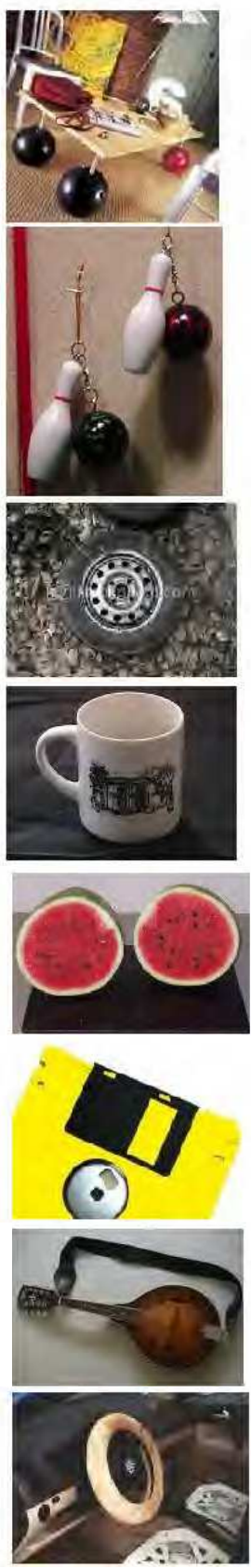

(b) Canny edge map
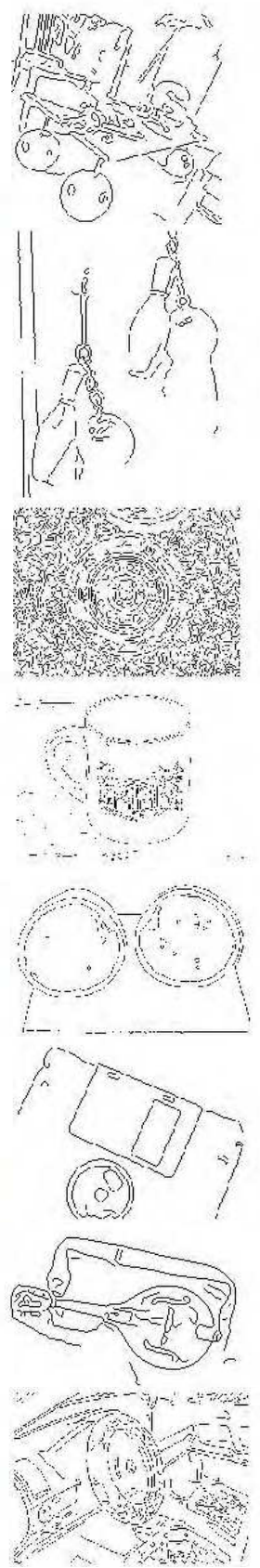

(c) Extracted edges
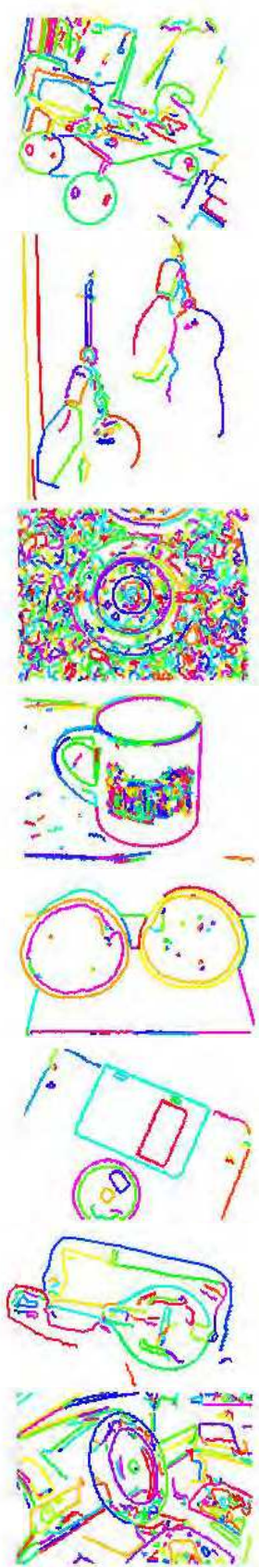

(d) Detected ellipses
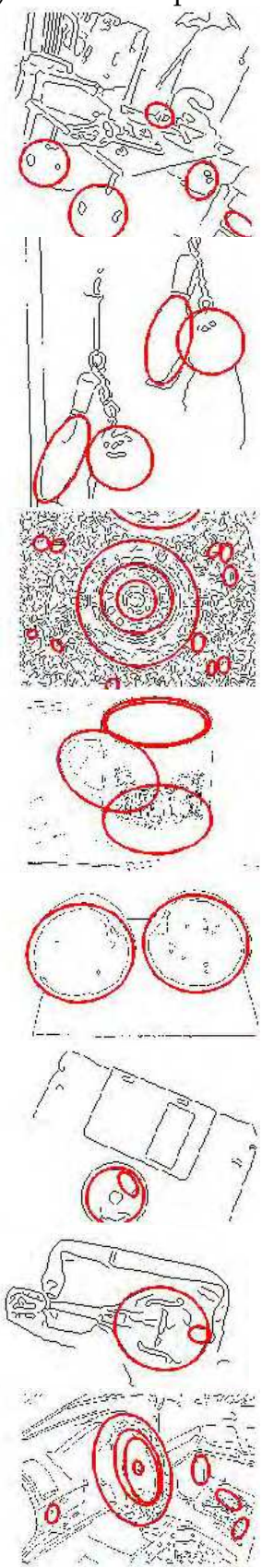

Fig. 13. Examples of real images and ellipse detection using the proposed method (continued). 

(a) Original Image
(b) Canny edge map
(c) Extracted edges
(d) Detected ellipses
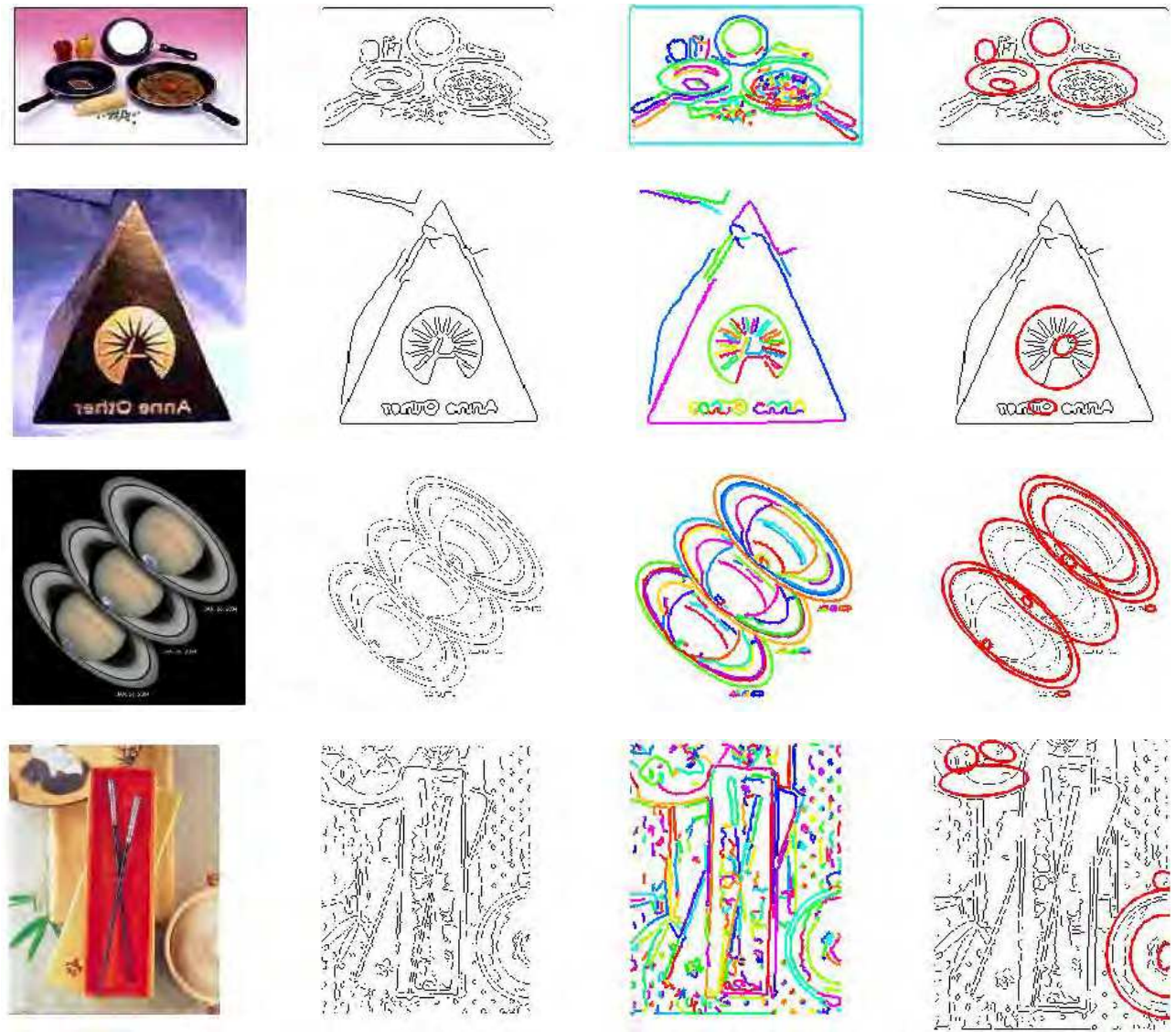

Fig. 14. Examples of real images and ellipse detection using the proposed method (continued).

\section{Conclusion}

The challenges in the problem of ellipse detection in real images are clearly identified. The need to use more selective ellipse detection methods, that can detect elliptic shapes with greater accuracy and reduce the false detections, is highlighted. A new unconstrained linearly computable least squares method is proposed. This method uses a linear matrix formulation and transfers the non-linearity of the ellipse detection problem to a new set of variables that are linked non-linearly to the geometric parameters of the ellipses. This method shows significantly better performance than the widely used non-linear constrained least squares formulation of Fitzgibbon (Fitzgibbon et al., 1999). The proposed method has lower false positive as well as false negative rates.

A hybrid ellipse detection method is also presented. This method uses various steps to deal with the various challenges of the problem of ellipse detection. Due to these sophisticated 
pre-processing, grouping, ellipse detection, and ellipse selection steps, the proposed method is highly selective and detects ellipses in various challenging scenario.

\section{References}

Aguado, A.S., Montiel, M.E. and Nixon, M.S., 1995. Ellipse detection via gradient direction in the Hough transform, Proceedings of the International Conference on Image Processing and its Applications, pp. 375-378.

Aguado, A.S., Montiel, M.E. and Nixon, M.S., 1996. Improving parameter space decomposition for the generalised Hough transform, Proceedings of the IEEE International Conference on Image Processing, pp. 627-630.

Ahn, S.J. and Rauh, W., 1999. Geometric least squares fitting of circle and ellipse. International Journal of Pattern Recognition and Artificial Intelligence, 13(7): 987996.

Ahn, S.J., Rauh, W., Cho, H.S. and Warnecke, H.J., 2002. Orthogonal distance fitting of implicit curves and surfaces. IEEE Transactions on Pattern Analysis and Machine Intelligence, 24(5): 620-638.

Ahn, S.J., Rauh, W. and Warnecke, H.J., 2001. Least-squares orthogonal distances fitting of circle, sphere, ellipse, hyperbola, and parabola. Pattern Recognition, 34(12): 22832303.

Anderson, I.M. and Bezdek, J.C., 1984. Curvature and tangential deflection of discrete arcs: a theory based on the commutator of scatter matrix pairs and its application to vertex detection in planar shape data. IEEE Transactions on Pattern Analysis and Machine Intelligence, PAMI-6(1): 27-40.

Antonaros, G.I. and Petrou, L.P., 2001. Real time map building by means of an ellipse spatial criterion and sensor-based localization for mobile robot. Journal of Intelligent and Robotic Systems: Theory and Applications, 30(4): 331-358.

Baeza-Yates, R. and Ribeiro-Neto, B., 1999. Modern Information Retrieval. ACM Press, Addison-Wesley, New York.

Basca, C.A., Talos, M. and Brad, R., 2005. Randomized hough transform for ellipse detection with result clustering, Proceedings of the International Conference on Computer as a Tool, Belgrade, SERBIA MONTENEG, pp. 1397-1400.

Belaroussi, R., Prevost, L. and Milgram, M., 2005. Classifier combination for face localization in color images, Lecture Notes in Computer Science, pp. 1043-1050.

Bell, A.A., Herberich, G., Meyer-Ebrecht, D., Bocking, A. and Aach, T., 2006. Segmentation and detection of nuclei in silver stained cell specimens for early cancer diagnosis, Proceedings of the International Conference on Image Processing, San Antonio, TX, pp.VI-49 - VI-52.

Bhowmick, P. and Bhattacharya, B.B., 2007. Fast polygonal approximation of digital curves using relaxed straightness properties. IEEE Transactions on Pattern Analysis and Machine Intelligence, 29(9): 1590-1602.

Burrill, J.R., Wang, S.X., Barrow, A., Friedman, M. and Soffen, M., 1996. Model-based matching using elliptical features, Proceedings of SPIE - The International Society for Optical Engineering, pp. 87-97. 
Carmona-Poyato, A., Madrid-Cuevas, F.J., Medina-Carnicer, R. and Muñoz-Salinas, R., 2010. Polygonal approximation of digital planar curves through break point suppression. Pattern Recognition, 43(1): 14-25.

Cazals, F. and Pouget, M., 2005. Estimating differential quantities using polynomial fitting of osculating jets. Computer Aided Geometric Design, 22(2): 121-146.

Cheng, Y.C., 2006. The distinctiveness of a curve in a parameterized neighborhood: Extraction and applications. IEEE Transactions on Pattern Analysis and Machine Intelligence, 28(8): 1215-1222.

Chia, A.Y.-S., Rahardja, S., Rajan, D. and Leung, M.K.H., 2011. A Split and Merge Based Ellipse Detector with Self-Correcting Capability. IEEE Transactions on Image Processing, 20(7): 1991 - 2006.

Chia, A.Y.S., Rahardja, S., Rajan, D. and Leung, M.K.H., 2009. Structural descriptors for category level object detection. IEEE Transactions on Multimedia, 11(8): 1407-1421.

Chia, A.Y.S., Rajan, D., Leung, M.K.H. and Rahardja, S., 2008. A split and merge based ellipse detector, Proceedings of the IEEE International Conference on Image Processing, San Diego, CA, pp. 3212-3215.

Dijkers, J.J. et al., 2005. Segmentation and size measurement of polyps in CT colonography, Lecture Notes in Computer Science, pp. 712-719.

Ellis, T., Abbood, A. and Brillault, B., 1992. Ellipse detection and matching with uncertainty. Image and Vision Computing, 10(5): 271-276.

Fernandes, A.M., 2009. Study on the Automatic Recognition of Oceanic Eddies in Satellite Images by Ellipse Center Detection-The Iberian Coast Case. IEEE Transactions on Geoscience and Remote Sensing, 47(8): 2478-2491.

Feyaerts, F., Vanroose, P., Fransens, R. and Van Gool, L.J., 2001. Using shape to correct for observed non-uniform color in automated egg grading, Proceedings of SPIE - The International Society for Optical Engineering, pp. 93-101.

Fitzgibbon, A., Pilu, M. and Fisher, R.B., 1999. Direct least square fitting of ellipses. IEEE Transactions on Pattern Analysis and Machine Intelligence, 21(5): 476-480.

Foresti, G.L., 2002. Detecting elliptical structures in underwater images. Journal of Electronic Imaging, 11(1): 59-68.

Foresti, G.L., Micheloni, C. and Piciarelli, C., 2005. Detecting moving people in video streams. Pattern Recognition Letters, 26(14): 2232-2243.

Fu, G. and Huang, S., 1995. Computer-aided control in nonround process, Proceedings of SPIE - The International Society for Optical Engineering, pp. 184-189.

Goneid, A., ElGindi, S. and Sewisy, A., 1997. A method for the Hough Transform detection of circles and ellipses using a 1-dimensional array, Proceedings of the IEEE International Conference on Systems, Man, and Cybernetics, Orlando, Fl, pp. 31543157.

Griffin, G., Holub, A. and Perona, P., Caltech-256 object category database. California Institute of Technology, pp. http://authors.library.caltech.edu/7694.

Guil, N. and Zapata, E.L., 1997. Lower order circle and ellipse Hough transform. Pattern Recognition, 30(10): 1729-1744.

Hahn, K., Jung, S., Han, Y. and Hahn, H., 2008. A new algorithm for ellipse detection by curve segments. Pattern Recognition Letters, 29(13): 1836-1841. 
Harker, M., O'Leary, P. and Zsombor-Murray, P., 2008. Direct type-specific conic fitting and eigenvalue bias correction. Image and Vision Computing, 26(3): 372-381.

He, Z., Tan, T., Sun, Z. and Qiu, X., 2009. Toward accurate and fast iris segmentation for iris biometrics. IEEE Transactions on Pattern Analysis and Machine Intelligence, 31(9): 1670-1684.

Heikkila, J., 1998. Moment and curvature preserving technique for accurate ellipse boundary detection. In: A.K. Jain, S. Venkatesh and B.C. Lovell (Editors), Proceedings of the International Conference on Pattern Recognition, Brisbane, Australia, pp. 734-737.

Ho, C.T. and Chen, L.H., 1995. A fast ellipse/circle detector using geometric symmetry. Pattern Recognition, 28(1): 117-124.

Hua, C., Wu, H., Chen, Q. and Wada, T., 2007. Object tracking with target and background samples. IEICE Transactions on Information and Systems, E90-D(4): 766-774.

Hwang, S., Oh, J., Tavanapong, W., Wong, J. and De Groen, P.C., 2006. Polyp detection in colonoscopy video using elliptical shape feature, Proceedings of the International Conference on Image Processing, San Antonio, TX, pp. II-465 - II-468.

Iles, P.J., Brodland, G.W., Clausi, D.A. and Puddister, S.M., 2007. Estimation of cellular fabric in embryonic epithelia. Computer methods in biomechanics and biomedical engineering, 10(1): 75-84.

Illingworth, J. and Kittler, J., 1988. A survey of the hough transform. Computer Vision, Graphics and Image Processing, 44(1): 87-116.

Ji, G.-R., Lu, B.-L., Chen, X. and Wang, J., 1999. Object searching in scale-space, Proceedings of the IEEE International Conference on Systems, Man and Cybernetics, Tokyo, Japan, pp. I-565 - I-570.

Ji, Q. and Haralick, R.M., 2001. Error propagation for the Hough transform. Pattern Recognition Letters, 22(6-7): 813-823.

Kasemir, K.U. and Betzler, K., 2003. Detecting ellipses of limited eccentricity in images with high noise levels. Image and Vision Computing, 21(2): 221-227.

Kawaguchi, T. and Nagata, R., 1998a. Ellipse detection using a genetic algorithm. In: A.K. Jain, S. Venkatesh and B.C. Lovell (Editors), Proceedings of the International Conference on Pattern Recognition, Brisbane, Australia, pp. 141-145.

Kawaguchi, T. and Nagata, R.I., 1998b. Ellipse detection using grouping of edgels into linesupport regions, Proceedings of the IEEE International Conference on Image Processing, pp. 70-74.

Kayikcioglu, T., Gangal, A. and Ozer, M., 2000. Reconstructing ellipsoids from three projection contours. Pattern Recognition Letters, 21(11): 959-968.

Kim, E., Haseyama, M. and Kitajima, H., 2002. Fast and Robust Ellipse Extraction from Complicated Images, Proceedings of the International Conference on Information Technology and Applications, pp. 357-362.

Kovesi, P.D., MATLAB and Octave Functions for Computer Vision and Image Processing, pp. http://www.csse.uwa.edu.au/ pk/Research/MatlabFns/index.html.

Kumar, N., Kohlbecher, S. and Schneider, E., 2009. A novel approach to video-based pupil tracking. 2009 IEEE International Conference on Systems, Man and Cybernetics, SMC 2009, San Antonio, TX, pp. 1255-1262. 
Kuno, Y., Okamoto, Y. and Okada, S., 1991. Robot vision using a feature search strategy generated from a 3-D object model. IEEE Transactions on Pattern Analysis and Machine Intelligence, 13(10): 1085-1097.

Liu, Y., Ikenaga, T. and Goto, S., 2007. Geometrical, physical and text/symbol analysis based approach of traffic sign detection system. IEICE Transactions on Information and Systems, E90-D(1): 208-216.

Lu, W., Tan, J. and Floyd, R.C., 2005. Fetal head detection and measurement in ultrasound images by a direct inverse randomized hough transform, Proceedings of the SPIE on Progress in Biomedical Optics and Imaging, pp. 715-722.

Lu, W. and Tan, J.L., 2008. Detection of incomplete ellipse in images with strong noise by iterative randomized Hough transform (IRHT). Pattern Recognition, 41(4): 12681279 .

Mai, F., Hung, Y.S., Zhong, H. and Sze, W.F., 2008. A hierarchical approach for fast and robust ellipse extraction. Pattern Recognition, 41(8): 2512-2524.

Maini, E.S., 2006. Enhanced direct least square fitting of ellipses. International Journal of Pattern Recognition and Artificial Intelligence, 20(6): 939-953.

Masood, A., 2008. Dominant point detection by reverse polygonization of digital curves. Image and Vision Computing, 26(5): 702-715.

Matas, J., Shao, Z. and Kittler, J., 1995. Estimation of curvature and tangent direction by median filtered differencing. Lecture Notes in Computer Science, 974: 83-88.

Matson, W.L., McKinstry, H.A., Johnson Jr, G.G., White, E.W. and McMillan, R.E., 1970. Computer processing of SEM images by contour analyses. Pattern Recognition, 2(4).

McLaughlin, R.A., 1998. Randomized Hough transform: Improved ellipse detection with comparison. Pattern Recognition Letters, 19(3-4): 299-305.

McLaughlin, R.A. and Alder, M.D., 1998. The hough transform versus the upwrite. IEEE Transactions on Pattern Analysis and Machine Intelligence, 20(4): 396-400.

Meer, P., Mintz, D., Rosenfeld, A. and Kim, D.Y., 1991. Robust regression methods for computer vision: A review. International Journal of Computer Vision, 6(1): 59-70.

O'Leary, P., Harker, M. and Zsombor-Murray, P., 2005. Direct and least square fitting of coupled geometric objects for metric vision, pp. 687-694.

Prasad, D.K. and Leung, M.K.H., 2012. Polygonal Representation of Digital Curves, Digital Image Processing, Stefan G. Stanciu (Editor), InTech, pp. 71-90.

Prasad, D.K., 2011. A geometric technique for tangent estimation for digital curves. ForseLab-11-001, Nanyang Technological University, Singapore.

Prasad, D.K., Gupta, R.K. and Leung, M.K.H., 2011a. An Error Bounded Tangent Estimator for Digitized Elliptic Curves, Lecture Notes in Computer Science. Lecture Notes in Computer Science. Springer Berlin / Heidelberg, pp. 272-283.

Prasad, D.K. and Leung, M.K.H., 2010a. An ellipse detection method for real images, 25th International Conference of Image and Vision Computing New Zealand (IVCNZ 2010), Queenstown, New Zealand.

Prasad, D.K. and Leung, M.K.H., 2010b. Error analysis of geometric ellipse detection methods due to quantization, Fourth Pacific-Rim Symposium on Image and Video Technology (PSIVT 2010), Singapore, pp. 58 - 63. 
Prasad, D.K. and Leung, M.K.H., 2010c. A hybrid approach for ellipse detection in real images. In: K. Jusoff and Y. Xie (Editors), 2nd International Conference on Digital Image Processing. SPIE, Singapore, pp. 75460I-6.

Prasad, D.K. and Leung, M.K.H., 2010d. Reliability/Precision Uncertainty in Shape Fitting Problems, IEEE International Conference on Image Processing, Hong Kong, pp. 4277-4280.

Prasad, D.K. and Leung, M.K.H., 2010e. Clustering of Ellipses based on their Distinctiveness: An aid to Ellipse Detection Algorithms, 3rd IEEE International Conference on Computer Science and Information Technology (ICCSIT 2010), Chengdu, China, pp. 292 - 297.

Prasad, D.K., Leung, M.K.H., Cho, S.Y. and Quek, C., 2011b. A parameter independent line fitting method, 1st IAPR Asian Conference on Pattern Recognition (ACPR 2011), Beijing, China, pp. 441-445.

Prasad, D.K. 2011c. Adaptive traffic signal control system with cloud computing based online learning, Eighth International Conference on Information, Communications, and Signal Processing (ICICS 2011), Singapore.

Princen, J., Illingworth, J. and Kittler, J., 1994. Hypothesis testing - a framework for analyzing and optimizing Hough transform performance. IEEE Transactions on Pattern Analysis and Machine Intelligence, 16(4): 329-341.

Procter, S. and Illingworth, J., 1994. A comparison of the randomised Hough transform and a genetic algorithm for ellipse extraction, Pattern Recognition in Practice IV. Elsevier, Amsterdam, pp. 449-460.

Qiao, Y. and Ong, S.H., 2007. Arc-based evaluation and detection of ellipses. Pattern Recognition, 40(7): 1990-2003.

Rosin, P.L., 1993a. Ellipse fitting by accumulating five-point fits. Pattern Recognition Letters, 14(8): 661-669.

Rosin, P.L., 1993b. A note on the least squares fitting of ellipses. Pattern Recognition Letters, 14(10): 799-808.

Rosin, P.L., 1996a. Analysing error of fit functions for ellipses. Pattern Recognition Letters, 17(14): 1461-1470.

Rosin, P.L., 1996b. Assessing error of fit functions for ellipses. Graphical Models and Image Processing, 58(5): 494-502.

Rosin, P.L. and West, G.A.W., 1992. Detection and verification of surfaces of revolution by perceptual grouping. Pattern Recognition Letters, 13(6): 453-461.

Rosin, P.L. and West, G.A.W., 1995. Nonparametric segmentation of curves into various representations. IEEE Transactions on Pattern Analysis and Machine Intelligence, 17(12): 1140-1153.

Salas, J., Avalos, W., CastaÃteda, R. and Maya, M., 2006. A machine-vision system to measure the parameters describing the performance of a Foucault pendulum. Machine Vision and Applications, 17(2): 133-138.

Ser, P.K. and Siu, W.C., 1995. Novel detection of conics using 2-D Hough planes, Proceedings of the IEE Vision, Image and Signal Processing, pp. 262-270.

Shen, Y., Yu, J. and Wang, Y., 2009. Fetal skull analysis in ultrasound images based on iterative randomized hough transform, Proceedings of the SPIE on Progress in Biomedical Optics and Imaging. 
Shih, F.Y., Cheng, S., Chuang, C.F. and Wang, P.S.P., 2008. Extracting faces and facial features from color images. International Journal of Pattern Recognition and Artificial Intelligence, 22(3): 515-534.

Smereka, M. and Glab, G., 2006. Detection of pathological cells in phase contrast cytological images, Lecture Notes in Computer Science, pp. 821-832.

Soetedjo, A. and Yamada, K., 2005. Fast and robust traffic sign detection, Proceedings of the IEEE International Conference on Systems, Man and Cybernetics, pp. 1341-1346.

Sood, V., John, B., Balasubramanian, R. and Tandon, A., 2005. Segmentation and tracking of mesoscale eddies in numeric ocean models, Proceedings of the International Conference on Image Processing, pp. 469-472.

Takimoto, H., Mitsukura, Y. and Akamatsu, N., 2004. Face Identification Based on Ellipse Parameter Independent of Varying Facial Pose and Lighting Condition, Lecture Notes in Computer Science, pp. 874-880.

Tang, C.Y., Chen, Z. and Hung, Y.P., 2000. Automatic detection and tracking of human heads using an active stereo vision system. International Journal of Pattern Recognition and Artificial Intelligence, 14(2): 137-166.

Tsuji, H. and Matsumoto, F., 1978. Detection of ellipses by a modified Hough transformation. IEEE Transactions on Computers, C-27(8): 777-781.

Wang, C., Newman, T.S. and Cao, C., 2007. New hypothesis distinctiveness measure for better ellipse extraction, Lecture Notes in Computer Science, pp. 176-186.

Wang, M., Yang, J. and Liu, W., 2006. Extraction of line and rounded objects from underwater images. Journal of Harbin Institute of Technology (New Series), 13(5): 613-620.

Weisstein, E.W., CRC concise encyclopedia of mathematics, 2. CRC Press, Florida.

Worring, M. and Smeulders, A.W.M., 1993. Digital Curvature Estimation. Computer Vision and Image Understanding, 58(3): 366-382.

Wu, W.Y. and Wang, M.J.J., 1993. Elliptical object detection by using its geometric properties. Pattern Recognition, 26(10): 1499-1509.

Yip, R.K.K., Tam, P.K.S. and Leung, D.N.K., 1992. Modification of hough transform for circles and ellipses detection using a 2-dimensional array. Pattern Recognition, 25(9): 1007-1022.

Yip, R.K.K., Tam, P.K.S. and Leung, D.N.K., 1995. Modification of hough transform for object recognition using a 2-dimensional array. Pattern Recognition, 28(11): 17331744.

Yuasa, M., Yamaguchi, O. and Fukui, K., 2004. Precise Pupil Contour Detection Based on Minimizing the Energy of Pattern and Edge. IEICE Transactions on Information and Systems, E87-D(1): 105-112.

Yuen, H.K., Illingworth, J. and Kittler, J., 1989. Detecting partially occluded ellipses using the Hough transform. Image and Vision Computing, 7(1): 31-37.

Zaim, A., Quweider, M., Scargle, J., Iglesias, J. and Tang, R., 2006. A robust and accurate segmentation of iris images using optimal partitioning, Proceedings of the International Conference on Pattern Recognition, pp. 578-581.

Zhang, J.Z., Wu, Q.M.J. and Gruver, W.A., 2003. Active head tracking based on chromatic shape fitting. International Journal of Pattern Recognition and Artificial Intelligence, 17(4): 529-544. 
Zhang, S.C. and Liu, Z.Q., 2005. A robust, real-time ellipse detector. Pattern Recognition, 38(2): 273-287.

Zhong, B., Ma, K.K. and Liao, W., 2009. Scale-space behavior of planar-curve corners. IEEE Transactions on Pattern Analysis and Machine Intelligence, 31(8): 1517-1524.

Zhou, J. and Shen, J., 2003. Ellipse detection and phase demodulation for wood grain orientation measurement based on the tracheid effect. Optics and Lasers in Engineering, 39(1): 73-89.

Zhou, Q.C., Liu, H.S., Kondrashkov, V.V., Li, G.D. and Lin, Y.H., 2009. Ellipse evolving common reflection point velocity analysis and its application to oil and gas detection. Journal of Geophysics and Engineering, 6(1): 53-60. 


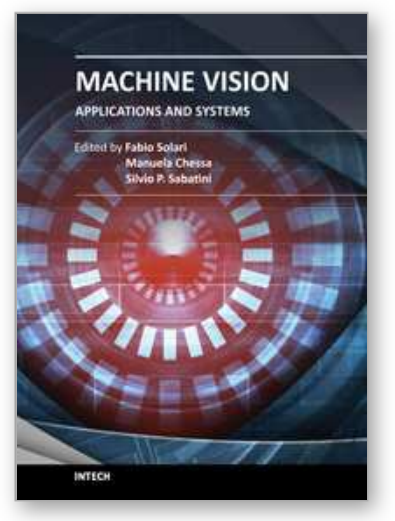

\author{
Machine Vision - Applications and Systems \\ Edited by Dr. Fabio Solari
}

ISBN 978-953-51-0373-8

Hard cover, 272 pages

Publisher InTech

Published online 23, March, 2012

Published in print edition March, 2012

Vision plays a fundamental role for living beings by allowing them to interact with the environment in an effective and efficient way. The ultimate goal of Machine Vision is to endow artificial systems with adequate capabilities to cope with not a priori predetermined situations. To this end, we have to take into account the computing constraints of the hosting architectures and the specifications of the tasks to be accomplished, to continuously adapt and optimize the visual processing techniques. Nevertheless, by exploiting the low?cost computational power of off?the?shell computing devices, Machine Vision is not limited any more to industrial environments, where situations and tasks are simplified and very specific, but it is now pervasive to support system solutions of everyday life problems.

\title{
How to reference
}

In order to correctly reference this scholarly work, feel free to copy and paste the following:

Dilip K. Prasad and Maylor K.H. Leung (2012). Methods for Ellipse Detection from Edge Maps of Real Images, Machine Vision - Applications and Systems, Dr. Fabio Solari (Ed.), ISBN: 978-953-51-0373-8, InTech, Available from: http://www.intechopen.com/books/machine-vision-applications-and-systems/methods-forellipse-detection-from-edge-maps-of-real-images

\section{INTECH}

open science | open minds

\section{InTech Europe}

University Campus STeP Ri

Slavka Krautzeka 83/A

51000 Rijeka, Croatia

Phone: +385 (51) 770447

Fax: +385 (51) 686166

www.intechopen.com

\section{InTech China}

Unit 405, Office Block, Hotel Equatorial Shanghai

No.65, Yan An Road (West), Shanghai, 200040, China

中国上海市延安西路65号上海国际贵都大饭店办公楼405单元

Phone: +86-21-62489820

Fax: $+86-21-62489821$ 
(C) 2012 The Author(s). Licensee IntechOpen. This is an open access article distributed under the terms of the Creative Commons Attribution 3.0 License, which permits unrestricted use, distribution, and reproduction in any medium, provided the original work is properly cited. 